Hier können Sie CME-Punkte sammeln a) für die Pflichtfortbildung aller Vertragsärzte und b) für freiwillige Fortbildungszertifikate, die viele Landesärztekammern anbieten.

Die Multiple-Choice-Fragen beziehen sich auf den vorangegangenen Fortbildungsbeitrag (Seiten 29-35). Die Antworten ergeben sich z. T. aus dem Text, z. T. beruhen sie auf medizinischem Basiswissen!

Wenn Sie 70\% der Fragen richtig beantworten, erhalten Sie 2, bei 100\% 3 CME-Punkte.

Teilnahmeschluss: 11. Januar 2009 (www.cme-punkt.de) bzw. 25. September 2008 (Einsendung per Brief).
CME-Herausgeber- und Review-Board:

Dr. H. J. K. Barwitz, Prof. Dr. A. Berghaus, Prof. Dr.

Dr. h.c. Th. Brandt, Prof. Dr. W. G. Daniel, Prof. Dr.

W. Eisenmenger, Prof. Dr. K. Friese, Prof. Dr. H. S.

Füessl, Prof. Dr. B. Göke, Prof. Dr. R. Gradinger, Prof. Dr. H. Holzgreve, Prof. Dr. A. Imdahl, Prof.

Dr. K.-W. Jauch, Prof. Dr. K. Krüger, Prof. Dr. H.-J.

Möller, Prof. Dr. D. Reinhardt, Prof. Dr. Dr. h.c. Th.

Ruzicka, Prof. Dr. Ch. Stief, U. Weigeldt.

\title{
Fragen zum Thema „Hyperthyreose“
}

\section{- 1. Die Inzidenz der manifesten Hyperthy- reose nimmt im Alter zu und beträgt in Deutschland etwa}

A $0,7 \%$.

B $1,4 \%$

C $4,2 \%$.

D $6,3 \%$.

E $20 \%$.

- 2. Die Labordiagnose ist im Alter schwieriger als bei Jüngeren, da ältere Patienten

A häufiger Kontrastmitteluntersuchungen bekommen.

B häufiger ein erniedrigtes TBG infolge Lebersynthesestörungen haben.

C aufgrund von Multimorbidität ein erniedrigtes TSH im Rahmen eines NTIS (Non thyroid illness syndrom) haben.

D häufiger Schilddrüsenhormone einnehmen.

E häufiger Knotenstrumen haben.

\section{- 3. Welche Symptome treten bei älteren} Patienten mit Hyperthyreose signifikant häufiger auf als bei jüngeren?

A Müdigkeit und Leistungsschwäche

B Tremor und vermehrtes Schwitzen

C Vorhofflimmern und Gewichtsverlust

D Obstipation und Apathie

E Wärmeintoleranz und Polydipsie

- 4. Eine 86-jährige Patientin mit COPD, Herzinsuffizienz und kompensierter Niereninsuffizienz (Kreatinin 3,2 mg\%) leidet an einer Hyperthyreose (TSH 0,01 $\mu \mathrm{U} / \mathrm{ml}$, fT4 1,8 ng/dl, fT3 5,2 pg/ml) auf dem Boden einer multifokalen Autonomie. Welche Therapieentscheidung würden Sie treffen?

\section{A Radiojodtherapie}

B Radiojodtherapie nach vorheriger thyreostatischer Behandlung, nach Normalisierung der peripheren Schilddrüsenwerte

C Thyreostatische Behandlung bis zur Erreichung der Euthyreose, evtl. langzeitige thyreostatische Behandlung

D Thyreoidektomie ohne Vorbehandlung

E Thyreoidektomie nach thyreostatischer Vorbehandlung

\section{- 5. Welche Maßnahme treffen Sie bei} einer 76-jährigen Patientin, die wegen einer immunogenen Hyperthyreose nach 9-monatiger Therapie mit Carbimazol ein allergisches Exanthem entwickelt?

A Carbimazol absetzen und mit Propycil weiterbehandeln

B Genaue Anamnese erheben, insbesondere nach anderen Allergenen fahnden

C Carbimazol absetzen und Radiojodtherapie vorschlagen

D Carbimazol absetzen und den weiteren Verlauf abwarten

E Eine Thyreoidektomie vorschlagen

\section{-6. Welches ist die häufigste Ursache der} Hyperthyreose in Deutschland?

A Morbus Basedow

B Hashimoto-Thyreoiditis

C Hyperthyreosis factitia

D Schilddrüsenautonomie

E Thyreoiditis de Quervain

-7. Welcher immunologische Befund ist mit einem Morbus Basedow assoziiert?
A TPO-Antikörper
B TSH-Rezeptor-Antikörper
C DNA-Antikörper
D Thyreoglobulin-Antikörper
E Antinukleäre Antikörper

\section{- 8. Was ist die häufigste extrathyreoidale} Manifestation des Morbus Basedow?

A Akropachie

B Prätibiales Myxödem

C Osteoporose

D Akromegalie

E Endokrine Orbitopathie

\section{-9. Beim Vergleich von Autonomie und} M. Basedow (M.B.) trifft welche Aussage zu?

A Von M.B. sind vor allem ältere Menschen betroffen.

B Bei der Autonomie treten keine extrathyreoidalen Manifestationen auf.

C Die Autonomie ist seltener als der M.B.

D Die Klinik beim M.B. beginnt schleichend, bei der Autonomie akut.

E Die Autonomie ist selten mit einer Struma assoziiert.

-10. Welcher Parameter sollte zur Therapiekontrolle unter thyreostatischer Therapie in den ersten Monaten beobachtet werden?

A Hertel-Index

B TRAK

C TSH

D fT3, fT4

E Schilddrüsenvolumen

\section{ANTWORTFORMULAR}

Mit Einreichen dieses Fragebogens erkläre ich mich damit einverstanden, dass die angegebenen Daten zum Zweck der Teilnahmebestätigung gespeichert und bei erfolgreicher Teilnahme an den Einheitlichen Informationsverteiler (EIV) der Ärztekammern weitergegeben werden.

Wichtiger Hinweis:

Eine Auswertung ist künftig nur noch möglich, wenn Sie Ihre EFN auf dem Antwortformular angeben! Nähere Hinweise hierzu unter: www.cme-punkt.de/faq.html
„Hyperthyreose“

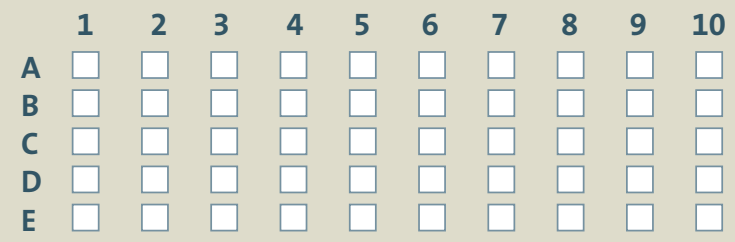

Bitte kreuzen Sie pro Frage je eine Antwort deutlich an.
Und so kommen Sie zu Ihren Punkten:

Teilnahme im Internet: unter www.cme-punkt.de. Dort führen wir für Sie ein elektronisches Punktekonto.

Teilnahme per Brief: Fragebogen ausfüllen und mit frankiertem Rückumschlag an: Urban \& Vogel GmbH CME MMW-Fortschr. Med. Postfach 81664 München. 